\title{
Cumulative reaction probabilities: A comparison between quasiclassical and quantum mechanical results
}

\author{
F. J. Aoiz ${ }^{\text {a) }}$ \\ Departamento de Química Física, Facultad de Química, Universidad Complutense, 28040 Madrid, Spain \\ and Department of Chemistry, The Physical and Theoretical Chemistry Laboratory, \\ University of Oxford, South Parks Road, Oxford OX1 3QZ, United Kingdom \\ M. Brouard ${ }^{\text {b) }}$ and C. J. Eyles \\ Department of Chemistry, The Physical and Theoretical Chemistry Laboratory, University of Oxford, \\ South Parks Road, Oxford OX1 3QZ, United Kingdom \\ J. F. Castillo \\ Departamento de Química Física, Facultad de Química, Universidad Complutense, 28040 Madrid, Spain \\ V. Sáez Rábanos ${ }^{\mathrm{c})}$ \\ Departamento de Qumica y Bioqumica, ETS Ingenieros de Montes, Universidad Politécnica, \\ 28040 Madrid, Spain
}

(Received 7 June 2006; accepted 17 August 2006; published online 11 October 2006)

This article presents a quasiclassical trajectory (QCT) method for determining the cumulative reaction probability (CRP) as a function of the total energy. The method proposed is based on a discrete sampling using integer values of the total and orbital angular momentum quantum numbers for each trajectory and on the development of equations that have a clear counterpart in the quantum mechanical $(\mathrm{QM})$ case. The calculations comprise cumulative reaction probabilities at a given total angular momentum $J$, as well as those summed over $J$. The latter are used to compute QCT rate constants. The method is illustrated by comparing QCT and exact QM results for the $\mathrm{H}_{+} \mathrm{H}_{2}, \mathrm{H}$ $+\mathrm{D}_{2}, \mathrm{D}+\mathrm{H}_{2}$, and $\mathrm{H}+\mathrm{HD}$ reactions. The agreement between QCT and QM results is very good, with small discrepancies between the two data sets indicating some genuine quantum effects. The most important of these involves the value of the CRP at low energies which, due to the absence of tunneling, is lower in the QCT calculations, causing the corresponding rate constants to be smaller. The second is the steplike structure that is clearly displayed in the QM CRP for $J=0$, which is much smoother in the corresponding QCT results. However, when the QCT density of reactive states, i.e., the derivatives of the QCT CRP with respect to the energy, is calculated, a succession of maxima and minima is obtained which roughly resembles those found in the QM calculations, although the latter are considerably sharper. The analysis of the broad peaks in the QCT density of reactive states indicates that the distributions of collision times associated with the maxima are somewhat broader, with a tail extending to larger collision times, than those associated with the minima. In addition, the QM and QCT dynamics of the isotopic variants mentioned above are compared in the light of their CRPs. Issues such as the compliance of the QCT CRP with the law of microscopic reversibility, as well as the similarity between the CRPs for ortho and para species in the QM and QCT cases, are also addressed. () 2006 American Institute of Physics. [DOI: 10.1063/1.2353837]

\section{INTRODUCTION}

The cumulative reaction probability (CRP), defined as the sum of reaction probabilities summed over all initial and final states, provides a link between scattering theory and the transition state formulation of the rate constant. ${ }^{1-7}$ Indeed, the expressions of the microcanonical and canonical rate constants in terms of the CRP are formally identical to those obtained within the framework of transition state theory (TST). In particular, the microcanonical rate constant is given by

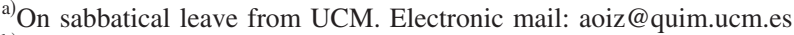

${ }^{b)}$ Electronic mail: mark.brouard@chemistry.oxford.ac.uk

${ }^{c)}$ Electronic mail:v.saez@upm.es
}

$$
k(E)=\frac{C_{r}(E)}{h \rho^{R}(E)},
$$

where $C_{r}(E)$ is the CRP (Ref. 8) at total energy $E, h$ the Planck constant, and $\rho^{R}(E)$ the reactant density of states per unit energy. Similarly, the canonical rate constant $k(T)$ is given by the Boltzmann average of $k(E)$ :

$$
k(T)=\int_{0}^{\infty} k(E) P(E ; T) d E=\frac{1}{Q_{R}(T)} \int k(E) \rho^{R}(E) e^{-\beta E} d E,
$$

where $\beta=1 / k_{B} T$ and $Q_{R}(T)$ is the total (translational and internal) partition function of the reagents. Clearly, knowledge of the CRP provides all the dynamical information nec- 
essary for the exact calculation of canonical and microcanonical rate constants.

The term CRP seems to have been coined by Miller in 1975 (Ref. 1) in the context of a quantum mechanical (QM) and semiclassical version of TST, and its concept was subsequently developed in a series of articles. ${ }^{2-4,9,10}$ The CRP may be interpreted qualitatively as the number of reactive reactant states at energy $E$. In some versions of TST (those which quantize the motion orthogonal to the reaction coordinate and neglect tunneling and recrossing effects) the CRP is predicted to increase in stepwise unit increments at the energy levels of the activated complex, and thus, within TST, the CRP may then be interpreted precisely and equivalently as the number of reactive reactant states or the number of transition states at energy $E$. The exact CRP, on the other hand, increases in smooth "steps" that can take any value between 0 and 1 . Although the exact CRP only shows steplike structure if the vibrational motion during the reaction is to some extent adiabatic, the interpretation of the CRP as the number of reactive reactant states is qualitatively correct and remains very useful.

More recently, Truhlar and co-workers have demonstrated further the importance of the CRP. ${ }^{5-7,11}$ Although the $\mathrm{CRP}$ is an averaged magnitude, its dependence on energy contains valuable information about the quantized spectrum of the transition state and the global dynamics of the reaction. Truhlar and co-workers showed that for the $\mathrm{H}_{3}$ system, the derivative of the CRP with respect to energy, the density of reactive states (DRS), displays pronounced peaks that can be attributed to quantized transition states or quantum bottlenecks. They were able to fit the exact QM results with a model based on an inverted parabolic barrier for each of these quantum states and adjustable transmission coefficients to account for recrossing. In this way, a set of spectroscopic constants of the activated complex was determined, and the various peaks in the DRS were assigned to different quantum transition states. The latter could be characterized by three quantum numbers, corresponding to the symmetric stretch, the bend, and the vibrational angular momentum, $\left[v_{\mathrm{ss}}, v_{b}^{\Omega}\right]$, of a short-lived linear complex. These quantized transition states are well approximated by locally vibrationally adiabatic dynamical bottlenecks that control the overall reactivity. ${ }^{11-13}$

The CRP is not necessarily a monotonically increasing function of the energy. Especially for total angular momentum $J=0$, the CRP may show other quantum effects related in some cases with resonances, either of the Feschbach type or shape-type resonances associated with van der Waals wells in either the entrance or the exit channel. ${ }^{14-19}$

For some time the usefulness of the CRP was limited by the fact that its accurate determination required a full scattering calculation of the $S$ matrix. An alternative to circumvent this problem is the quantum formulation of the rate constant in terms of the flux autocorrelation function. ${ }^{9,20-24}$ Specifically, the flux operator can be used to calculate CRPs or flux autocorrelation functions, from which rate constants may be obtained. This formulation can be applied using a variety of approaches, either within the time-independent or the time-dependent formalism, and an increasingly complex series of chemical reactions has thereby been successfully modeled. ${ }^{25-29}$ These techniques are particularly promising since they scale with the number of dimensions much more slowly than conventional scattering approaches.

In spite of the importance of the CRP, it has seldom been considered in quasiclassical trajectory (QCT) calculations. Chapman et al. ${ }^{10}$ reported collinear and three-dimensional (3D) calculations of CRPs for the $\mathrm{H}+\mathrm{H}_{2}$ reaction using classical trajectories. In order to compare with the results from classical transition state theory, these calculations were not quasiclassical but involved a purely classical microcanonical sampling of the initial conditions. While TST was found to describe the reactivity in the threshold region very accurately, as the energy was increased the dynamical calculations yielded reaction probabilities and microcanonical cross sections significantly lower than those obtained using TST. This effect can be ascribed to the neglect of recrossing trajectories in TST. Note that recrossing was found to play a much less important role when the reaction is studied in full dimensionality. The dependence of the CRP on total energy calculated in this way ${ }^{3,10}$ is remarkably similar to that obtained later using exact QM methods and to those presented in this work.

Much more recently, Lin et al. ${ }^{18,19}$ compared QM and QCT reaction probabilities and CRPs for $J=0$ for the direct, forward reaction between $\mathrm{O}\left({ }^{3} P\right)$ and $\mathrm{HCl}$ and its reverse, $\mathrm{Cl}+\mathrm{OH}$. Although they found relatively good agreement between the two theoretical approaches, especially for the latter reaction, the CRPs for the direct and reverse reactions derived using QCT methods were not identical, as required by the principle of microscopic reversibility. This was attributed to the much larger extent to which the direct reaction is vibrationally adiabatic in classical mechanics, relative to the reverse reaction. Apart from this study, to the best of our knowledge there have been no QCT calculations of CRPs. In a recent article, some of the present authors ${ }^{30}$ developed a QCT method to determine reaction probabilities as a function of $J$ for any value of the rotational angular momentum quantum number. The present calculation of the CRP constitutes a natural extension of that work. We present a procedure based on a discrete sampling of integer quantum number values for the various angular momenta involved in the determination of the CRP. In this sense, the method is truly quasiclassical and the corresponding QCT expressions have clear QM counterparts.

The QCT method proposed in this work is not meant to constitute an alternative to rigorous, fully converged QM calculations, which are nowadays routine for direct, collinearly dominated reactions. It is well known that classical mechanics, even in its most sophisticated quasiclassical version, fails to describe the dynamics in the threshold region, which is strongly dominated by tunneling. Given the importance of that energy region for the determination of thermal rate constants, this is a major shortcoming of any classical mechanic method. Nevertheless, the availability of an efficient QCT procedure may be useful in cases where a full QM calculation, including all possible $J$ values, is still prohibitively computationally expensive. Under these circumstances, the comparison between QCT and QM CRPs for low $J$ values 
could be extremely useful in validating the QCT approximation. Note also that, unlike fully converged QM calculations, ${ }^{32}$ the computational effort required to study a reaction such as $\mathrm{H}^{+}+\mathrm{H}_{2}$ using QCT methods is only slightly greater than that needed for the title reaction.

This article is organized as follows. In Sec. II we present expressions for the QCT CRP as a function of the total energy $E$ and total angular momentum quantum number $J$, while in Sec. III we outline the characteristics of the QM calculations. In order to compare QCT and QM results, the obvious choice of system is the $\mathrm{H}_{3}$ exchange reaction, for which very accurate theoretical data are available (for a recent review, see Ref. 31), and in Sec. IV results are shown for the following isotopic variants:

$$
\begin{aligned}
& \mathrm{H}+\mathrm{H}_{2} \rightarrow \mathrm{H}_{2}+\mathrm{H}, \\
& \mathrm{H}+\mathrm{D}_{2} \rightarrow \mathrm{D}+\mathrm{HD}, \\
& \mathrm{D}+\mathrm{H}_{2} \rightarrow \mathrm{H}+\mathrm{HD}, \\
& \mathrm{H}+\mathrm{HD} \rightarrow \mathrm{H}+\mathrm{DH}, \\
& \mathrm{H}+\mathrm{HD} \rightarrow \mathrm{D}+\mathrm{H}_{2} .
\end{aligned}
$$

Fully converged QM calculations have been performed for all these reactions, and $J$ specific and total CRPs are thus available. This comprehensive set of exact QM results provides a sound benchmark to test the corresponding QCT results and to establish its limitations. QM and QCT results are compared for CRPs at fixed $J$, for total CRPs, and for rate constants. Of particular interest is the investigation of the behavior of the DRS to test if some remnant of the QM bottleneck peak structure is found in the QCT results, and the primary focus of Sec. V is a detailed comparison between QCT and exact QM results for the DRS. We conclude in Sec. VI with a brief summary of the main results of this work. The practical details of the QCT calculation of CRPs by scanning the energy continuously are presented in the Appendix.

\section{GENERAL EXPRESSIONS AND QCT METHOD}

In terms of the reaction probabilities $P_{v j}^{J}(E)$ summed over product states, at a given total angular momentum quantum number $J$ and specific initial vibrational and rotational state $(v, j)$, the cumulative reaction probability can be written as

$$
C_{r}^{J}(E)=\sum_{v, j}[2 \min (J, j)+1] P_{v j}^{J}(E),
$$

where $C_{r}^{J}(E)$ is the CRP (Ref. 8) at a given $J$, and the sum comprises all the reagent states energetically open at a total energy $E$.

The expression for the reaction probability from a given $v, j$ initial state, summed over all $v^{\prime}, j^{\prime}$ final states, can be written in the orbital angular momentum and helicity representations as

$$
\begin{aligned}
P_{v j}^{J}(E) & =\frac{1}{2 \min (J, j)+1} \sum_{l=|J-j|}^{J+j} \sum_{v^{\prime} j^{\prime} l^{\prime}} P_{v^{\prime} j^{\prime} l^{\prime} \leftarrow v j l}^{J}(E) \\
& =\frac{1}{2 \min (J, j)+1} \sum_{K=-\min (J, j)}^{\min (J, j)} \sum_{v^{\prime} j^{\prime} K^{\prime}} P_{v^{\prime} j^{\prime} K^{\prime} \leftarrow v j K}^{J}(E),
\end{aligned}
$$

where $l\left(l^{\prime}\right)$ and $K\left(K^{\prime}\right)$ are the quantum numbers for the reactant (product) orbital angular momentum and the projection of $J$ onto the body fixed axis, respectively.

In $\mathrm{QM}$, the state-to-state reaction probability can be written in terms of the elements of the scattering matrix $S_{\alpha^{\prime} v^{\prime} j^{\prime} l^{\prime} 1 v j l}^{J,}$

$$
P_{v^{\prime} j^{\prime} l^{\prime} \leftarrow v j l}^{J}(E)=\sum_{P} \sum_{\alpha^{\prime} \neq 1}\left|S_{\alpha^{\prime} v^{\prime} j^{\prime} l^{\prime} 1 v j l}^{J P}\right|^{2},
$$

where the sums in Eq. (10) run over the triatomic parity $P$ and over the relevant product arrangement channels, $\alpha^{\prime} \neq 1$.

The corresponding QCT expression to Eq. (10) summed over final states is given by

$$
P_{v j l}^{J}(E)=\sum_{v^{\prime} j^{\prime} l^{\prime}} P_{v^{\prime} j^{\prime} l^{\prime} \leftarrow v j l}^{J}(E)=\frac{N_{r}(J, l ; E, v, j)}{N(J, l ; E, v, j)},
$$

where $N_{r}(J, l ; E, v, j)$ and $N(J, l ; E, v, j)$ are the number of reactive and the total number of trajectories, respectively, for given values of $J$ and $l$ and initial $v, j$ state, at a total energy E. $P_{v j l}^{J}(E)$ is the joint, quantal, or quasiclassical reaction probability, which depends on $J$ and $l$ for a given $v, j$ state and total energy $E .^{30}$

Following Ref. 30, the QCT method we propose considers discrete, integer values of $J$ and $l$ as well as $j$. For each trajectory with a given value of $J$ and energy $E, l$ is uniformly sampled in the interval $|J-j| \leqslant l \leqslant J+j$ using only integer values. With this sampling the reaction probability is given by

$$
P_{v j}^{J}(E)=\frac{N_{r}(v, j ; J, E)}{N(v, j ; J, E)},
$$

where $N_{r}(v, j ; J, E)$ is the number of reactive trajectories and $N(v, j ; J, E)$ the total number of trajectories, reactive or not, calculated for a single $v, j$ initial state, with specific values of $J$ and $E$. It is important to emphasize at this point that Eq. (12) holds only if $l$ is sampled uniformly. Substituting Eq. (12) into Eq. (8) yields the QCT expression for the CRP at fixed $E$ and $J$,

$$
C_{r}^{J}(E)=\sum_{v, j}[2 \min (J, j)+1] \frac{N_{r}(v, j ; J, E)}{N(v, j ; J, E)} .
$$

In order to carry out the calculation of $C_{r}^{J}(E)$, it is necessary to first count the number of reagent quantum states with energy below $E, n(E)$. Then, for every trajectory, one of these $n(E)$ states is chosen randomly with the same probability. For a sufficiently large number of trajectories, all energetically accessible $v, j$ states are equally sampled, and $N(v, j ; E, J) \approx N_{\alpha}$. If the total number of trajectories, including all initial $v, j$ states, for a given value of $J$ and $E$ is 
$N(J ; E)$, it becomes evident that $N_{\alpha}=N(J ; E) / n(E)$. Substituting this expression into Eq. (13) yields

$$
C_{r}^{J}(E)=\frac{n(E)}{N(J ; E)} \sum_{v, j}[2 \min (J, j)+1] N_{r}(v, j ; J, E) .
$$

For $J=0$, the expression is particularly simple, $C_{r}^{J=0}(E)$ $=n(E) N_{r}(0 ; E) / N(0 ; E)$. Apart from the selection of a particular initial state $v, j$, the rest of the sampling procedure for each trajectory has been explained in detail in Appendix B of Ref. 30.

The derivative of the CRP with respect to $E, \rho_{r}^{J}(E)$, is the DRS. This function has been extensively used ${ }^{5,6,11}$ and has proven to be most invaluable for the investigation of quantum bottlenecks.

The QCT expression of total CRP is given by

$$
\begin{aligned}
C_{r}(E) & =\sum_{J=0}^{J_{\max }}(2 J+1) C_{r}^{J}(E) \\
& =\sum_{J=0}^{J_{\max }} \sum_{v, j}[2 \min (J, j)+1](2 J+1) n(E) \frac{N_{r}(v, j ; J, E)}{N(J ; E)},
\end{aligned}
$$

where the sum over $J$ extends to the maximum $J$ value leading to reaction, $J_{\max }$, at energy $E$, and the sampling of each of the $n(E)$ energetically accessible states of the reagents is done with equal probability. Given the larger weights carried by higher $J$ values, it is more efficient to sample $J$ for each trajectory proportional to the number of projections, $2 J+1$. In practice, for each trajectory, $J$ is sampled according to $J$ $=\operatorname{int}\left[\xi^{1 / 2}\left(J_{\max }+1\right)\right]$, where $\xi$ is a random number in the $[0,1]$ interval, and int means to take the integer part of the expression. Once a $J$ number has been chosen, the orbital angular momentum quantum number is sampled uniformly for each trajectory within the interval $[|J-j|, J+j] .{ }^{30}$ Under these circumstances, the total CRP is given by

$$
\begin{aligned}
C_{r}(E)= & \frac{\left(J_{\max }+1\right)^{2}}{N(E)} n(E) \\
& \times \sum_{v, J} \sum_{J=0}^{J_{\max }}[2 \min (J, j)+1] N_{r}(v, j, J ; E),
\end{aligned}
$$

where $N(E)$ is the total number of trajectories run at a given total energy, and $N(J ; E)=(2 J+1) N(E) /\left(J_{\max }+1\right)^{2}$.

Instead of using discrete values of the total energy in the calculations, it is also possible to sample the total energy continuously and uniformly in the desired range $\left[E_{1}, E_{2}\right]$. The analysis of the results can be made using a series expansion in Legendre polynomials, as described in the Appendix.

In terms of the CRP, the thermal rate constant is given by

$$
k(T)=\frac{\int_{0}^{\infty} C_{r}(E) \exp \left(-E / k_{B} T\right) d E}{h \Phi_{\mathrm{rel}}(T) Q_{v, j}^{B C}(T)},
$$

where $\Phi_{\text {rel }}(T)$ is the translational partition function for the relative motion,

$$
\Phi_{\mathrm{rel}}(T)=\left(\frac{2 \pi \mu k_{B} T}{h^{2}}\right)^{3 / 2}
$$

and $Q_{v, j}^{B C}(T)$ is the rovibrational partition function of the reagents. When the QM or QCT $C_{r}(E)$ are given in a series of discrete $E$ values, we have found that the best method is to make an interpolation of the integrand, $C_{r}(E) \exp \left(-E / k_{B} T\right)$, in order to carry out the integral in Eq. (18). An alternative method, useful when trajectories are calculated by varying the energy continuously, is given in the Appendix.

For reactions with homonuclear diatomic molecules, the nuclear spin statistics has to be taken into account. The expression of the total CRP is in this case ${ }^{34}$

$$
C_{r}(E)=\sum_{T}(2 T+1) C_{r}^{T}(E),
$$

where $T$ is the molecular nuclear spin quantum number, i.e., for $\mathrm{H}_{2}\left(I=\frac{1}{2}\right) T=0,1$; for $\mathrm{D}_{2}(I=1) T=0,1,2$. For $p-\mathrm{H}_{2} T$ $=0$ (symmetric functions under permutation of the atoms), whereas for $o-\mathrm{H}_{2} T=1$ (antisymmetric under spatial permutation). Thereby for $\mathrm{H}+\mathrm{H}_{2}$ and $\mathrm{D}+\mathrm{H}_{2}$, the para and ortho CRPs are multiplied by $I(2 I+1)$ and $(I+1)(2 I+1)$, respectively. Similarly, for $\mathrm{H}+\mathrm{D}_{2}$ the symmetric functions are given by $T=0,2$ (ortho), with multiplicity $(2 I+1)(I+1)=6$, and the antisymmetric functions (para) are given by $T=1$, with multiplicity $I(2 I+1)=3$. As will be discussed in Sec. V, for the $\mathrm{H}_{3}$ system and its isotopic variants $C_{r}^{p}(E) \approx C_{r}^{o}(E)$.

Collision times $\tau$ were also calculated in this work for $J=0$. The collision time is the time that the three atoms spend close together without possible assignment to any of the three collision channels. When the three atoms are sufficiently close to each other, the potential is generally distorted with respect to its asymptotic behavior. This generally occurs when the initial $R$ and final $R^{\prime}$ Jacobi distances (defined from the atom to the center of mass of the diatomic) are less than two distance parameters $R_{\mathrm{int}}$ and $R_{\mathrm{int}}^{\prime}$, that define the strong interaction region. Their values depend on the reaction and are determined by following the time evolution of the interatomic distances and potential energy for a significant number of trajectories. In the present case, $R_{\text {int }}=R_{\text {int }}^{\prime}=2.1 \AA$. The collision times were calculated directly, recording the times when $R$ was first $\leqslant 2.1 \AA$ and when $R^{\prime}$ became $\geqslant 2.1 \AA$. The difference between these times gives the collision time.

All the calculations presented here were performed in the BKMP2 potential energy surface (PES). ${ }^{35}$ The integration step in the trajectory calculations was $5 \times 10^{-2} \mathrm{fs}$, ensuring an energy conservation better than 1 in $10^{5}$. The initial rovibrational energies were calculated semiclassically for all the states using the asymptotic diatomic potential energy of the PES, and their values are coincident with the exact QM ones within four significant figures. For the calculations using a discrete grid of total energies, batches of $5 \times 10^{4}-1 \times 10^{5}$ trajectories were calculated at each energy and $J$. Calculations of the total CRP were performed with a grid of energies with a spacing of $5 \times 10^{-3} \mathrm{eV}$ between 0.5 and $1.8 \mathrm{eV}$. For those calculations in which the energy was scanned continuously (see the Appendix), batches of trajectories of $5 \times 10^{5}$ $-2 \times 10^{6}$ were run for each individual $J$. For the analogous calculation of the total CRP, the number of trajectories was 
$2 \times 10^{6}$ for each of the isotopic variants studied. Overall the number of calculated trajectories relevant to the present work was of the order of $3 \times 10^{7}$. As a result of the large number of trajectories used to determine the $C_{r}^{J}(E)$, the resulting Monte Carlo uncertainties are of the order of $0.3 \%-1.5 \%$.

\section{QUANTUM MECHANICAL METHOD}

In order to compare with exact QM results and to assess the validity of the QCT calculations, we have made use of the extensive QM calculations for a series of isotopic variants of the $\mathrm{H}+\mathrm{H}_{2}$ reaction. These calculations were used in a previous article ${ }^{36}$ to compare accurate $\mathrm{QM}$ rate constants and kinetic isotope effects with those obtained with the quantum instanton approximation. The methodology of these calculations is based on the accurate determination of the cumulative reaction probabilities, and has been described extensively in the literature. ${ }^{1,33,34}$ Thus only a brief outline will be given in this section, along with the details of the specific calculations.

In the present work, CRPs have been calculated for all $J$ up to $J_{\max }$, including all the projections in $K$ and $K^{\prime}$ up to maximum values of $K=\min \left(J, j, K_{\max }\right)$ and $K^{\prime}$ $=\min \left(J, j^{\prime}, K_{\max }\right) . K_{\max }$ values were chosen such that for the highest energy calculated, for a given isotopic variant of the reaction, contributions from higher $K\left(K^{\prime}\right)$ are practically negligible. The QM reactive scattering matrix has been calculated using a coupled-channel hyperspherical coordinate method of Skouteries et al. ${ }^{37}$ Converged reaction probabilities and CRPs for the $\mathrm{H}+p-\mathrm{H}_{2}$ reaction on the BKMP2 PES were calculated for total angular momenta $J=0-35$ using a basis set including all $\mathrm{H}+p-\mathrm{H}_{2}$ and $\mathrm{HH}+\mathrm{H}$ channels with diatomic energy levels up to $E_{\max } \leqslant 2.6 \mathrm{eV}$ and rotational quantum numbers up to $j_{\max } \leqslant 15$. For this isotopic variant, a total of 70 energies has been calculated for the reaction, between 0.271 and $1.651 \mathrm{eV}$. Note that only reactions with $p$ $-\mathrm{H}_{2}$ have been considered because the results for $p-\mathrm{H}_{2}$ and $o-\mathrm{H}_{2}$ are practically identical up to energies $\approx 1.5 \mathrm{eV}$. It must be stressed that this is the only approximation used, and that the CRPs have been calculated for all partial waves $J$ $\in\left[0, J_{\max }\right]$. For the H+HD reaction the CRPs were calculated for $J=0-28$ using a basis set with $E_{\max } \leqslant 2.6 \mathrm{eV}$ and $j_{\max } \leqslant 16$. Using these basis sets, it was found that the CRPs were converged to better than $1 \%$ for $J=0$. For $J>0$, angular basis functions with helicities up to $K_{\max }=7$ for the reactant and product arrangements were retained. We have checked that for $J=12$ the CRPs are converged to $1.0 \%$ at the highest energy with the above parameters.

In addition, extra calculations of the CRP for $J=0-5$ for $\mathrm{D}+\mathrm{H}_{2}$ and $\mathrm{H}+\mathrm{D}_{2}$ were carried out including ortho and para states in an extended range of energies up to $2.25 \mathrm{eV}$, in order to compare the CRPs for the two diatomic parities. In both cases $E_{\max }=3.0 \mathrm{eV}$, and a rovibrational basis with $v_{\max }=6$ and $j_{\max }=30$ was employed, together with 300 integration sectors, and a maximum hyper-radius of $11 \AA$, at energy intervals of $0.02 \mathrm{eV}$.

QM calculations were also carried out with a fictitious

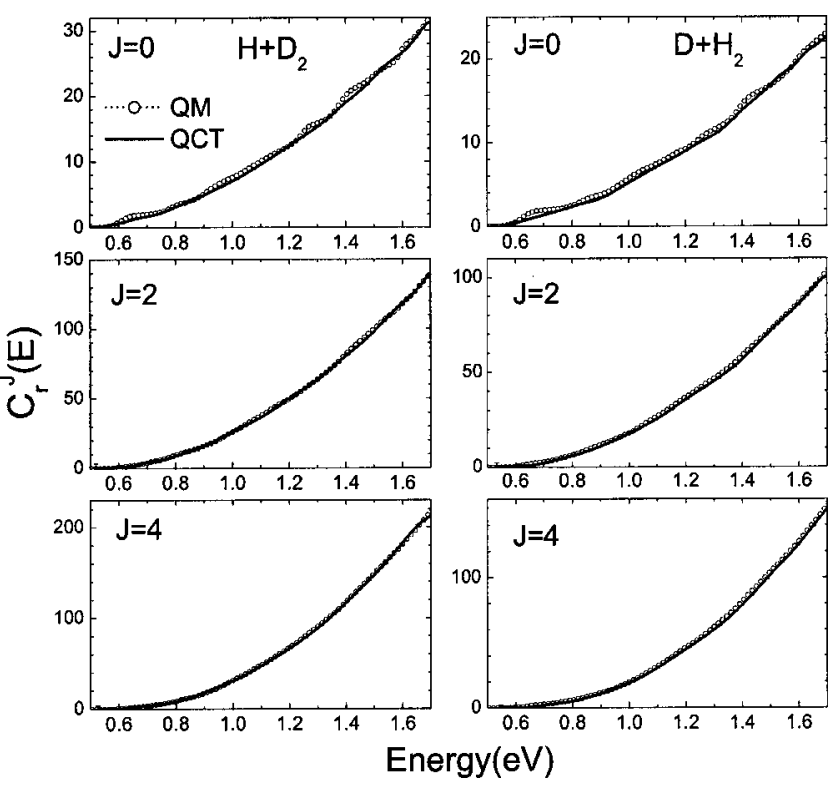

FIG. 1. Comparison of QM (open circles, dashed line) and QCT (solid line) cumulative reaction probabilities resolved in $J, C_{r}^{J}(E)$ (Ref. 8), as a function of the total energy (in $\mathrm{eV}$ ) for the $\mathrm{H}+\mathrm{D}_{2}$ (left panels) and $\mathrm{D}+\mathrm{H}_{2}$ (right panels) reactions at the indicated values of $J$. The $C_{r}^{J}(E)$ displayed here is given by the (unweighted) sum of the corresponding $C_{r}^{J}(E)$ for the ortho and para species. The error bars of the QCT CRP are equal or smaller than the width of the line.

Planck constant $h^{\prime}=h / \sqrt{10}$ for $\mathrm{H}+\mathrm{H}_{2}$ and $J=0$. For these calculations the rovibrational basis extends to $v_{\max }=15$ and $j_{\max }=60$.

\section{RESULTS}

We will first compare the QM and QCT $C_{r}^{J}(E)$ for the $\mathrm{H}+\mathrm{D}_{2} \rightarrow \mathrm{HD}+\mathrm{D}$ and $\mathrm{D}+\mathrm{H}_{2} \rightarrow \mathrm{HD}+\mathrm{H}$ reactions. Figure 1 displays these results for $J=0,2$, and 4. Unless otherwise stated, the CRPs shown here are the sum of those corresponding to the reaction with $p-\mathrm{H}_{2}\left(o-\mathrm{D}_{2}\right)$ and $o-\mathrm{H}_{2}\left(p-\mathrm{D}_{2}\right)$, that is, including even and odd $j$ states without any nuclear spin statistical weight. The QCT $C_{r}^{J}(E)$ are quite smooth, increasing monotonically with $E$. As can be seen, the agreement between the QCT and QM results is very good for all $J$ shown.

The main visible discrepancy between the two sets of results can be found in the steplike structure clearly observable in the QM $C_{r}^{J}(E)$ for $J=0$. This steplike behavior is reminiscent of the ideal quantized transition state thresholds that would appear in the absence of tunneling and recrossing. If transition state theory were an accurate description of the quantum dynamics, the $C_{r}^{J}(E)$ would increase in stepwise unit increments at the energy levels of the activated complex. This steplike pattern is sometimes associated with "classical" behavior (i.e., the absence of tunneling). However, a purely classical $C_{r}^{J}(E)$ would be completely smooth in the absence of quantization in reagents, transition state, or products. ${ }^{10}$ The steplike pattern implies at least a local adiabaticity in the region of the transition state. As $J$ increases, the agreement between QM and QCT results becomes better as the steplike structure smoothes out. As can be seen from the respective scales, the number of reactive states increases rapidly with $J$ 

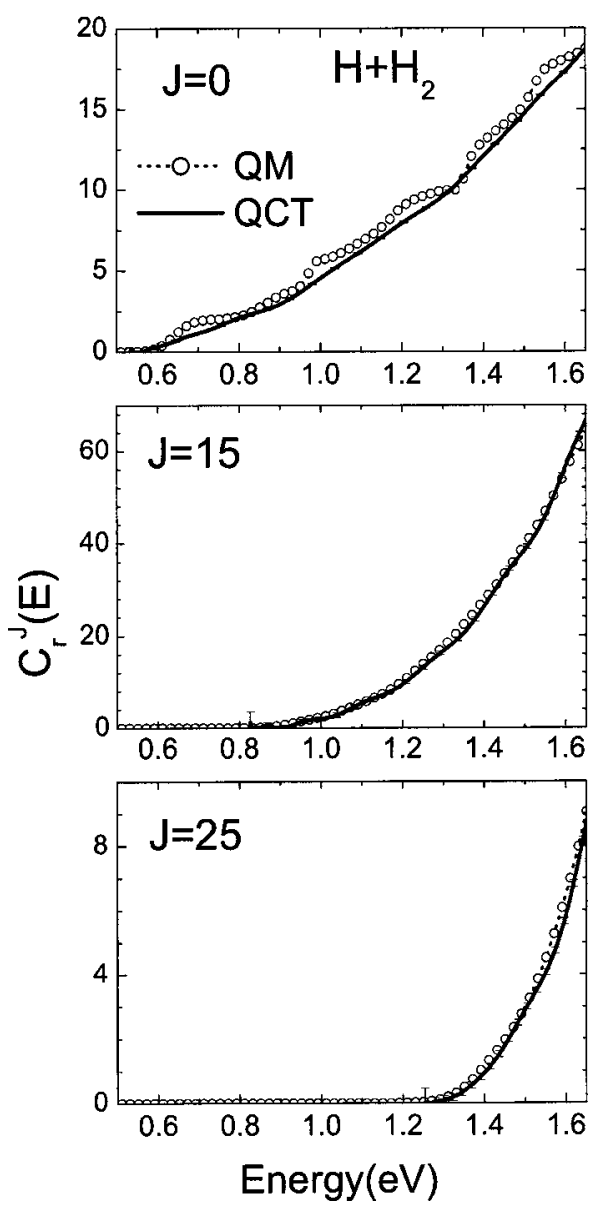

FIG. 2. Same as Fig. 1 but for $\mathrm{H}+\mathrm{H}_{2}$, showing results for $J=0,15,25$.

and the quantum bottlenecks associated with each of the steps in the CRP overlap, causing the disappearance of the structure observed for $J=0$. Although the agreement remains good at low energies on the scale employed in the figures, there is nonetheless a significant difference in behavior in the threshold region, as discussed in more detail below when the total CRPs are presented.

The comparison between the QM and QCT $C_{r}^{J}(E)$ for the $\mathrm{H}+\mathrm{H}_{2}$ reaction is shown in Fig. 2. The agreement between the two calculations is slightly worse for the $\mathrm{H}+\mathrm{H}_{2}$ reaction than the isotopic variants shown in Fig. 1. The steplike structure in $C_{r}^{J=0}(E)$ for this reaction (see Fig. 2) is clearly more pronounced than for the $\mathrm{H}+\mathrm{D}_{2}$ and $\mathrm{D}+\mathrm{H}_{2}$ reactions, and the difference with the QCT $C_{r}^{J=0}(E)$ is more noticeable. Since the rovibrational energy levels in $\mathrm{D}_{2}$ are more closely spaced than those of $\mathrm{H}_{2}$, the magnitude of $C_{r}^{J}(E)$ is considerably smaller in the $\mathrm{H}+\mathrm{H}_{2}$ and $\mathrm{D}+\mathrm{H}_{2}$ reactions than in the $\mathrm{HD}_{2}$ system.

In the case of the $\mathrm{H}+\mathrm{H}_{2}$ reaction, a larger range of $J$ states is shown in order to appreciate the increase in the total energy of the reaction threshold as $J$ increases. For a given energy, as $J$ increases larger values of $l$ become accessible, with the resulting increase of the centrifugal barrier and, consequently, the total energy threshold for reaction increases with $J$ as shown in Fig. 2. The change in the absolute value of the CRP with $J$ can be explained in similar terms. Initially, the degeneracy associated with each state, $2 \min (J, j)+1$,

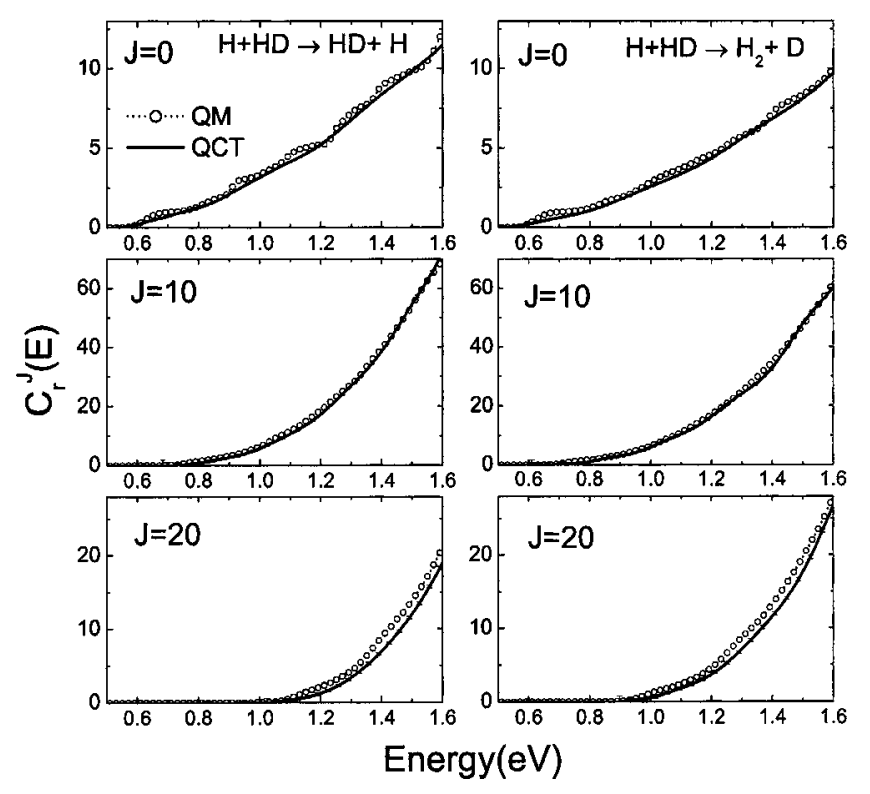

FIG. 3. Comparison of QM (open circles, dashed line) and QCT (solid line) $C_{r}^{J}(E)$ for $J=0,10$, and 20 for $\mathrm{H}+\mathrm{DH} \rightarrow \mathrm{HD}+\mathrm{H}$ (left) and $\mathrm{H}+\mathrm{HD} \rightarrow \mathrm{H}_{2}$ $+\mathrm{D}$ (right). The error bars of the QCT results are of the order of the width of the line.

causes the CRP to grow rapidly with $J$, but as larger values of $l$ become accessible, the reactivity (i.e., the number of reactive states) decreases. It is worth noticing that the reactivity of the $\mathrm{H}+\mathrm{H}_{2}$ system is smaller than for the $\mathrm{D}+\mathrm{H}_{2}$.

Figure 3 shows $C_{r}^{J}(E)(J=0,10,20)$ for the two reaction pathways of the $\mathrm{H}+\mathrm{HD}$ reaction, namely, the $\mathrm{H}$-atom exchange channel, leading to $\mathrm{HD}+\mathrm{H}$, and the $\mathrm{H}$-atom abstraction channel, leading to $\mathrm{H}_{2}+\mathrm{D}$. The CRPs at low $J$ values for the exchange $\mathrm{H}+\mathrm{DH} \rightarrow \mathrm{HD}+\mathrm{H}$ reaction are consistently higher than those for the $\mathrm{H}+\mathrm{HD} \rightarrow \mathrm{H}_{2}+\mathrm{D}$ abstraction reaction (see top panels of Fig. 3). However, with increasing $J$, the situation is reversed. At $J \geqslant 15$, the $C_{r}^{J}(E)$ for the abstraction channel is already larger at all total energies, with a lower threshold energy (see bottom panels of Fig. 3). At the highest calculated $J$ values (25-30), the difference becomes very important, with thresholds $0.2 \mathrm{eV}$ higher for the exchange $\mathrm{HD}+\mathrm{H}$ channel.

This behavior is a consequence of the asymmetry of the HD molecule with respect to its center of mass. Due to this asymmetry, two effects take place. On the one hand, collisions of the incoming $\mathrm{H}$ atom with the $\mathrm{H}$ side of the HD molecule atom can take place at higher $R$ values. Therefore, for a given collision energy and impact parameter, the centrifugal barrier will be smaller than in the case of the atom approach to the $\mathrm{H}$ part of molecule. On the other hand, the range of angles of attack leading to reaction will be more restricted for the $\mathrm{H}$ than for the $\mathrm{D}$ end of molecule. The net consequence of these opposite effects is that reactivity leading to HD formation (the exchange channel) will be clearly promoted for low values of $J$. However, as $J$ increases the centrifugal barrier becomes higher, and the preferred channel will be that yielding $\mathrm{H}_{2}+\mathrm{D}$ products. The influence of the mass asymmetry on the dynamics of this system has been thoroughly discussed in the literature (see for instance Refs. 

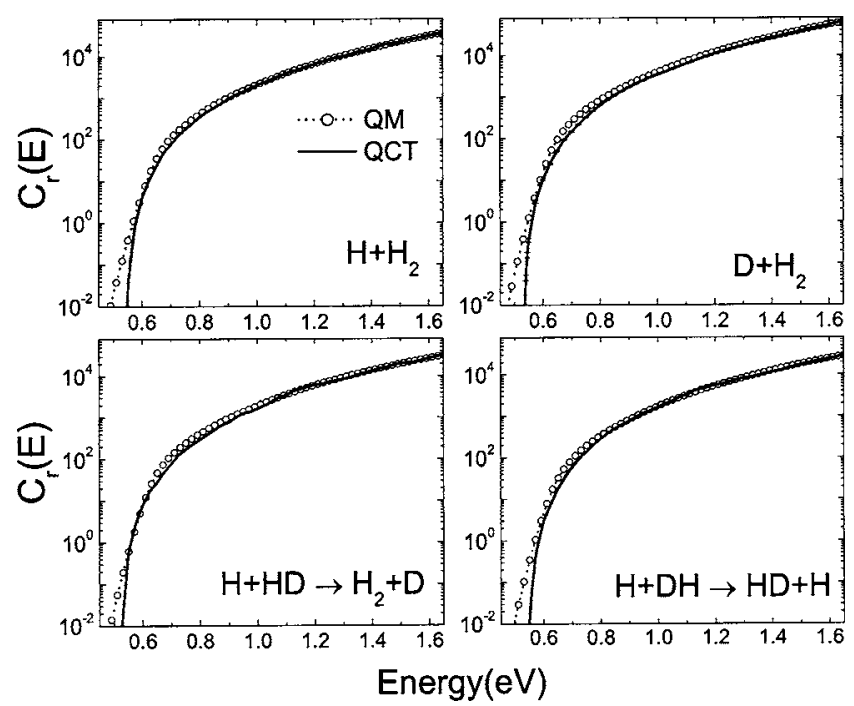

FIG. 4. Comparison of QM (open circles, dashed line) and QCT (solid line) total $C_{r}(E)$ as a function of total energy (incorporating $J=0-32$ for the QCT case) for $\mathrm{H}+\mathrm{H}_{2}$ (top left), $\mathrm{D}+\mathrm{H}_{2}$ (top right), $\mathrm{H}+\mathrm{HD} \rightarrow \mathrm{H}_{2}+\mathrm{D}$ (bottom left), and $\mathrm{H}+\mathrm{DH} \rightarrow \mathrm{HD}+\mathrm{H}$ (bottom right).

38 and 39 and references therein) but, to our knowledge, it has not been discussed in terms of CRP for various $J$ values.

It is noteworthy that the QM results invariably lie above the QCT data for the systems studied in the present work. This is most clearly seen for $J=0$, where the steps in the QM $C_{r}^{J}(E)$ are always above the QCT results, and in fact appear to sit on top of the smoother QCT curves. The effect is most marked in the threshold region, where classically there is not enough energy for reaction to take place, but is also observed as the energy increases sufficiently to access each new quantum bottleneck. One can picture the resulting $\mathrm{QM} C_{r}^{J}(E)$ as a series of contributions from individual bottleneck states, each with its own threshold that, when summed, gives rise to a relatively smooth steplike structure. The different behaviors seen in the QM and QCT calculations partly reflect the lack of tunneling in the QCT calculations, but is likely also to be due to the reduced tendency for recrossing of the transition state in the QM calculations. ${ }^{31}$ Interestingly, just below the energy of each new threshold, the QM and QCT CRPs tend to be in very good agreement. This implies that, in addition to the above factors, vibrational adiabaticity may also have a role to play in both the QM and the QCT calculations in the region of the onset of each new "quantum" bottleneck (the issue of vibrational adiabaticity is returned to in Sec. V). As $J$ increases, more states become allowed and their respective thresholds start to overlap, causing the disappearance of easily identifiable structures in the QM CRP, and thus the agreement between QM and QCT results improves. However, at high enough values of $J$ (see Figs. 2 and $3)$ the QM $C_{r}^{J}(E)$ are slightly higher than the QCT ones. In this case, it is tunneling through the combined potential and centrifugal barrier that enhances the reactivity in the QM case.

The total CRPs, defined by Eq. (15), are presented for reactions (3) and (5)-(7) in Fig. 4. The logarithmic scale is employed to emphasize the discrepancy between the QM and QCT results at low energies, and to show the variation of the

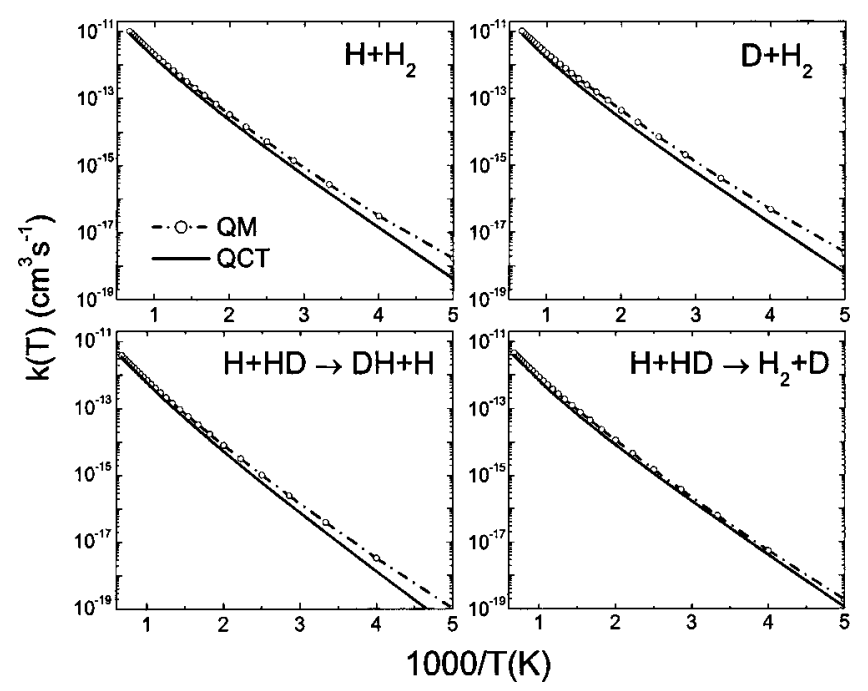

FIG. 5. Comparison of QM (open circles, dot-dash line) and QCT (solid line) $k(T)$ for $\mathrm{H}+\mathrm{H}_{2}$ (top left), $\mathrm{D}+\mathrm{H}_{2}$ (top right), $\mathrm{H}+\mathrm{DH} \rightarrow \mathrm{DH}+\mathrm{H}$ (bottom left), and $\mathrm{H}+\mathrm{HD} \rightarrow \mathrm{H}_{2}+\mathrm{D}$ (bottom right).

total CRP over seven orders of magnitude in this energy range. Although the absolute value of the QM CRP is very small for energies below $0.55 \mathrm{eV}$, the fact that classical threshold is relatively large for all these reactions has a decisive influence on the values of the rate constant (see below). The comparison with the QCT total CRP clearly indicates that below that energy threshold, all the reactivity is due to tunneling. Agreement between the QM and QCT results is generally good outside this region, becoming increasingly accurate at higher energies, although some discrepancies can also be observed at energies between 0.6 and $0.8 \mathrm{eV}$, where again the QM results are slightly above the QCT data. It seems that discrepancies between the QM and QCT CRPs above $0.6 \mathrm{eV}$ are most likely associated with tunneling through the combined potential and centrifugal barrier. As commented on above, the differences between the total CRPs of the exchange and abstraction channels for the $\mathrm{H}+\mathrm{HD}$ reaction are attributable to the mass asymmetry. Overall both QM and QCT predict higher reactivity for the latter channel.

Comparison of the QM and QCT rate constants $k(T)$ for reactions (3) and (5)-(7) is shown in Fig. 5. As expected, the QM rate constants are systematically higher than the QCT ones, the difference becoming more pronounced with decreasing temperature. At temperatures around $200 \mathrm{~K}$ these discrepancies can be associated almost entirely with QM tunneling below the barrier. The evident discrepancies between the QM and QCT rate constants observed at higher temperatures $(300-1000 \mathrm{~K})$ can be associated principally with the differences noted above in the total CRP in the energy range of $0.6-0.8 \mathrm{eV}$. These higher temperature discrepancies in the rate constants therefore arise principally from tunneling through the combined potential and centrifugal barrier at energies above threshold. The best agreement happens for the endothermic $\mathrm{H}+\mathrm{HD} \rightarrow \mathrm{H}_{2}+\mathrm{D}$ reaction. This can be attributed to the lack of quantization of the $\mathrm{H}_{2}$ product in the QCT calculations, which can therefore be formed below its zero point energy, and to some extent this compensates for the 

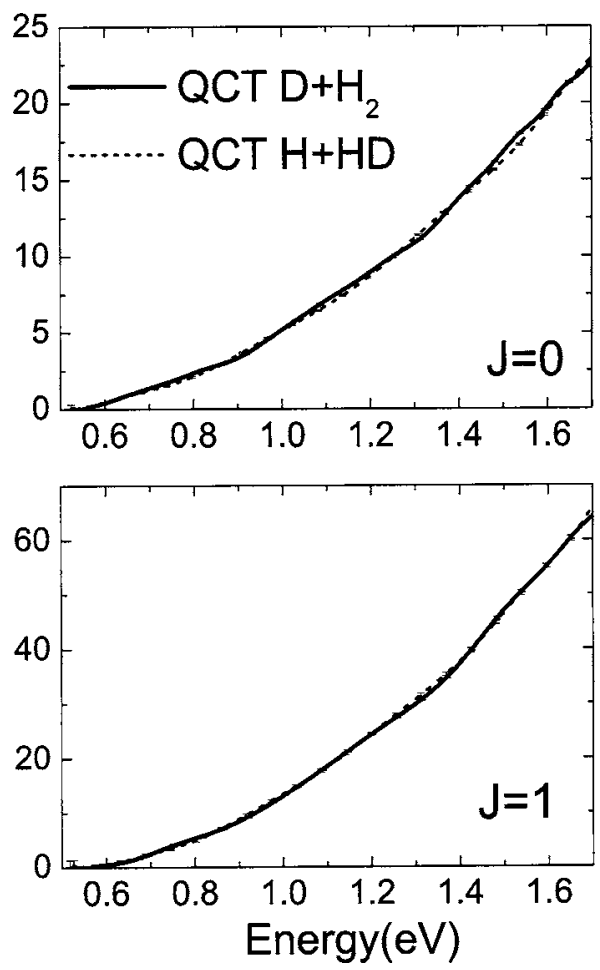

FIG. 6. Comparison of QCT $C_{r}^{J}(E)$ for $\mathrm{H}+\mathrm{HD} \rightarrow \mathrm{H}_{2}+\mathrm{D}$ multiplied by 2 (dashed line) and $C_{r}^{J}(E)$ for $\mathrm{D}+\mathrm{H}_{2}$ (solid line) as a function of total energy. QCT error bars are of the order of the width of the line.

absence of tunneling. This effect is reminiscent of that occurring in the QCT calculations of muonium with $\mathrm{H}_{2},{ }^{40}$ in which the violation of the zero point energy in the transition state and the products overcomes by far the neglect of tunneling, thereby yielding QCT values of $k(T)$ considerably higher than those obtained either experimentally or with accurate $\mathrm{QM}$ calculations. For the reverse $\mathrm{D}+\mathrm{H}_{2}$ reaction, the discrepancies between QM and QCT are more important. In this case, the reaction is exothermic (from the zero point energy) and the requirements for the HD zero point energy conservation are less stringent, given its lower value.

The results obtained here for $k(T)$ compare very well with previous $\mathrm{QM}$ and QCT calculations on the same or similar PESs. ${ }^{33,34,41-43}$ The comparison with the most recent theoretical calculations of $k(T)$ for the $\mathrm{H}+\mathrm{D}_{2}$ and $\mathrm{D}+\mathrm{H}_{2}$ by Mielke et $a l^{44}$ is also very good considering that in that study the effect of the Born-Oppenheimer diagonal correction was included in the calculations.

\section{DISCUSSION}

The invariance under time reversal establishes that the CRP for a given reaction and its reverse must be identical. While this is guaranteed in converged QM calculations, given the unitarity of the $S$ matrix, it is not necessarily the case in QCT calculations. Although microscopic reversibility is strictly adhered to by individual trajectories, this is not formally the case for the ensemble of trajectories, since only the reactant motions are pseudoquantized. In Fig. 6 the QCT CRPs for $J=0$ and 1 are shown for the $\mathrm{D}+\mathrm{H}_{2} \rightarrow \mathrm{H}+\mathrm{HD}$ (summing ortho and para contributions) and reverse $\mathrm{H}$ $+\mathrm{HD} \rightarrow \mathrm{H}_{2}+\mathrm{D}$ reactions. Note that the $C_{r}^{J}(E)$ for $\mathrm{H}+\mathrm{HD}$ has
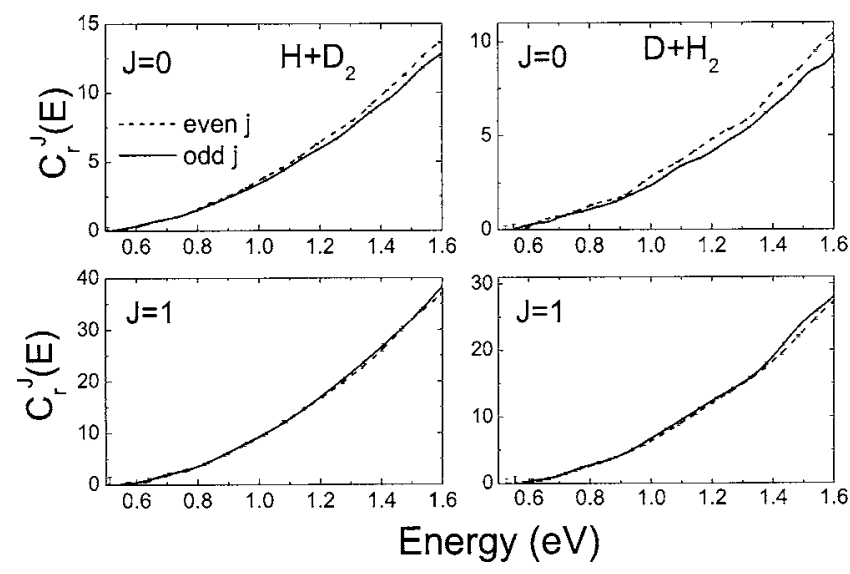

FIG. 7. Comparison of QCT $C_{r}^{J}(E)$ as a function of total energy for $\mathrm{H}+p$ $-\mathrm{D}_{2}$ (solid) and $\mathrm{H}+o-\mathrm{D}_{2}$ (dash) (left panels) and $\mathrm{D}+p-\mathrm{H}_{2}$ (dash) and $\mathrm{D}+o$ $-\mathrm{H}_{2}$ (solid) (right panels). Top panels: $J=0$, bottom panels: $J=1$.

been multiplied by 2 to account for the two indistinguishable arrangement channels for $\mathrm{D}+\mathrm{H}_{2}$. Rigorously speaking, one would equate the CRP of the $\mathrm{D}+p-\mathrm{H}_{2}$ divided by 2 to the CRP for the $\mathrm{H}+\mathrm{HD} \rightarrow p-\mathrm{H}_{2}+\mathrm{D}$, and similarly for the $o-\mathrm{H}_{2}$ species. In spite of small discrepancies, beyond the statistical uncertainties, between the two sets of calculations, the overall agreement is fairly good, both for the low $J$ data shown as well as at higher $J$. However, in the low energy region, due to the violation of the zero point energy in the endothermic $\mathrm{H}+\mathrm{HD} \rightarrow \mathrm{H}_{2}+\mathrm{D}$ reaction (see Sec. IV) the CRPs are systematically slightly higher for this reaction than for $\mathrm{D}+\mathrm{H}_{2}$. Although the differences are very small, it causes the total $C_{r}(E)$ to be greater at $E \leqslant 0.6 \mathrm{eV}$ for the latter reaction, and this is reflected in the values of its rate constant, which is in better agreement with the QM calculation than for any other isotopic variant examined here. The situation is analogous, although not so extreme, as that found for the $\mathrm{O}\left({ }^{3} \mathrm{P}\right)+\mathrm{HCl}$ and its reverse reaction, ${ }^{19}$ and in both cases the disagreement can be traced back to a smaller degree of adiabaticity of the reverse reactions.

It has been shown previously that the QM CRP for the hydrogen exchange reactions with $p-\mathrm{H}_{2}\left(o-\mathrm{D}_{2}\right)$ and $o-\mathrm{H}_{2}$ $\left(p-\mathrm{D}_{2}\right)$ are almost indistinguishable, and that the approximation of calculating just one diatomic parity is very accurate (to within three significant digits for energies below $1.5 \mathrm{eV}) .^{34,36,44}$ The corresponding QCT results are shown in Fig. 7. As can be seen, appreciable differences exist between the ortho and para QCT $C_{r}^{J}(E)$ for both the $\mathrm{D}+\mathrm{H}_{2}$ and $\mathrm{H}$ $+\mathrm{D}_{2}$ reactions, especially for $J=0$, although the differences become smaller as $J$ increases. Agreement is generally better for $\mathrm{H}+\mathrm{D}_{2}$ than for $\mathrm{D}+\mathrm{H}_{2}$. The $C_{r}^{J=0}(E)$ for $p-\mathrm{H}_{2}$ is consistently higher than that for $o-\mathrm{H}_{2}$ in the QCT case. By summing over all $J$, the difference between the CRP with even and odd diatomic parities becomes almost negligible but, in any case, we have used Eq. (20) to determine the $k(T)$.

As noted in Sec. I, Truhlar and co-workers ${ }^{5-7,11}$ have shown that the derivative of the CRP, the DRS, exhibits the type of behavior predicted by TST, with its most prominent features coinciding very well with vibrationally adiabatic transition state levels. These structures have been found in the QM derivative of the $C_{r}^{J}(E)$ presented in this work, and, 


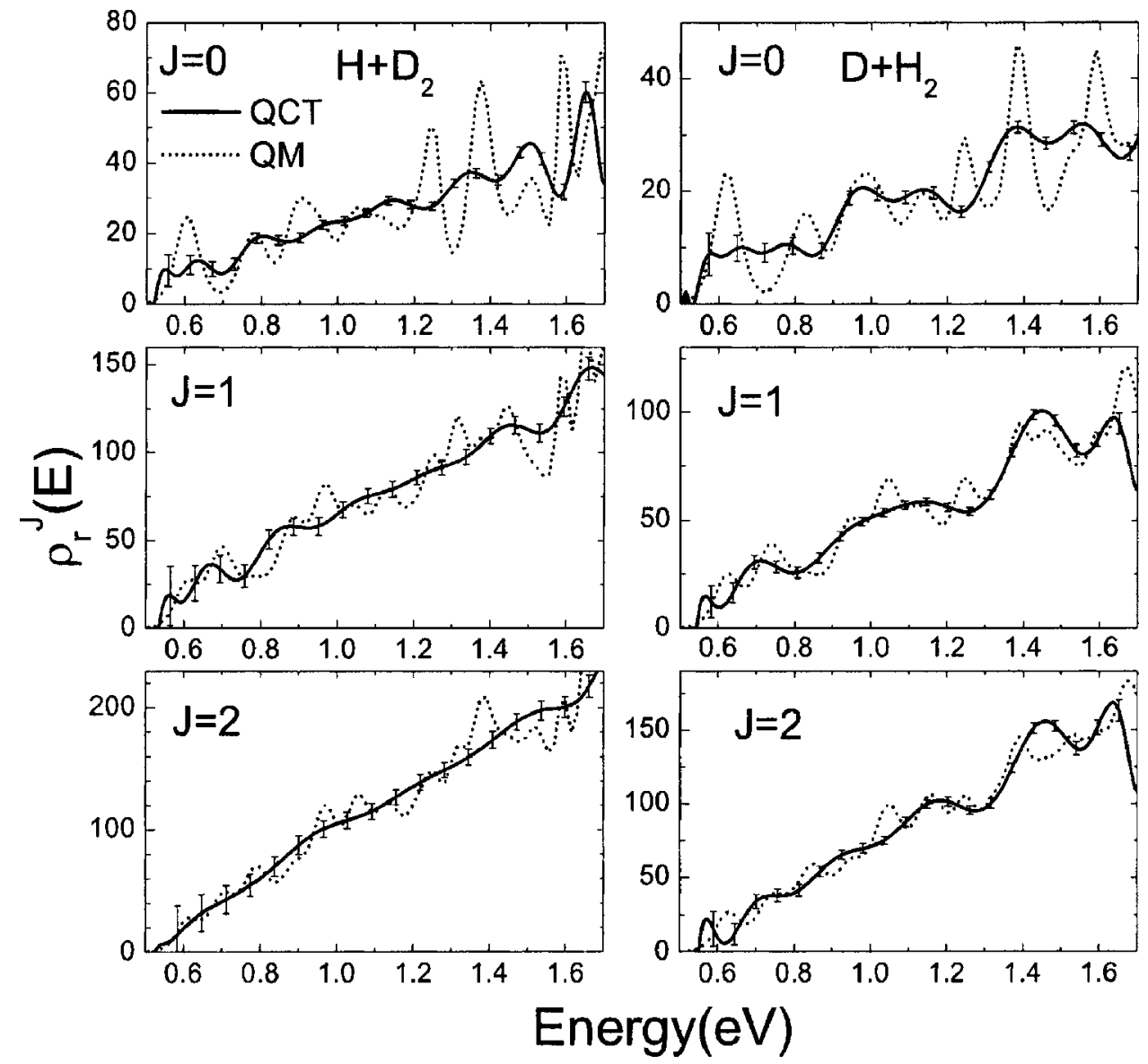

FIG. 8. Comparison of QM (dashed line) and QCT (solid line) densities of reactive states, $\rho_{r}^{J}(E)$, for $\mathrm{H}+\mathrm{D}_{2}$ (left) and $\mathrm{D}+\mathrm{H}_{2}$ (right) as a function of total energy at the indicated values of $J$. In both cases $\rho_{r}^{J}(E)$ is given by the unweighted sum of $\rho_{r}^{J, o}(E)+\rho_{r}^{J, p}(E)$. The error bars represent the statistical errors of the QCT $\rho_{r}^{J}(E)$.

apart from relatively small differences attributable to the use of a different PES, they coincide very well with those obtained in previous works. ${ }^{11,12}$ Subtle structures can also be detected in the QCT CRP, revealing slight deviations from a purely smooth behavior. It is instructive to compare the DRS obtained by differentiating the QCT CRP with those resulting from the exact QM calculations, particularly for low $J$, where the structures in the QM DRS are most pronounced. Such a comparison is shown in Fig. 8 for the $\mathrm{H}+\mathrm{D}_{2}$ and $\mathrm{D}+\mathrm{H}_{2}$ reactions with $J=0-2$. The QM and QCT DRSs are the sum of the para and ortho contributions in each case. The QCT DRS is found to have clear features, with a succession of maxima and minima that resembles those obtained in the QM case. As expected, the quantal results display much sharper peaks in the DRS, but the QCT results match the overall structure reasonably well, and there is some coincidence in the location of the maxima in many cases, something one might not expect if this structure were purely quantal in origin. To check the reliability of the QCT DRS, a sensitivity analysis of the general shape and position of the maxima was performed, both with discrete total energies and by scanning $E$ continuously and fitting the results using an expansion in Legendre polynomials (see the Appendix). Both procedures yielded almost identical results. The number of trajectories employed was $(3-4) \times 10^{6}$ for $J=0$ and about
$1 \times 10^{6}$ for the other $J$. The statistical uncertainties in the QCT DRS are well represented by the error bars shown in Fig. 8.

As $J$ increases, the QM DRS becomes smoother, and the various maxima overlap, although they still retain a clear structure. The QCT DRS seems to represent an average of the QM results over a certain range of energies. Nevertheless, some structure is still present, as can be seen for $J$ $=1,2$ in the $\mathrm{D}+\mathrm{H}_{2}$ reaction. Overall the agreement is quite good.

It is worth examining these features in more detail, separating out the contributions from ortho and para hydrogen to the $C_{r}^{J=0}(E)$. Recall that these contributions are not identical, at least in the QCT case. Figure 9 demonstrates the differences that exist between the QM and QCT $C_{r}^{J}(E)$ and $\rho_{r}^{J}(E)$ for $\mathrm{D}+o-\mathrm{H}_{2}$ and $\mathrm{D}+p-\mathrm{H}_{2}$ reactions. As mentioned above, the QM CRPs for both reactions are practically identical up to $E \approx 1.5 \mathrm{eV}$, and indeed their respective $\rho_{r}^{J}(E)$ below that energy are also indistinguishable within the scale of the plot. However, it is apparent that there is a significant difference between the QM DRSs for the ortho and para species at higher energies. For $p-\mathrm{H}_{2}$, the spectral assignment was carried out by Chatfield et al. ${ }^{11}$ on the Liu-Siegbahn-TruhlarHorowitz (LSTH) potential energy surface up to $1.6 \mathrm{eV}$, and that assignment is also valid here. However, the differences 

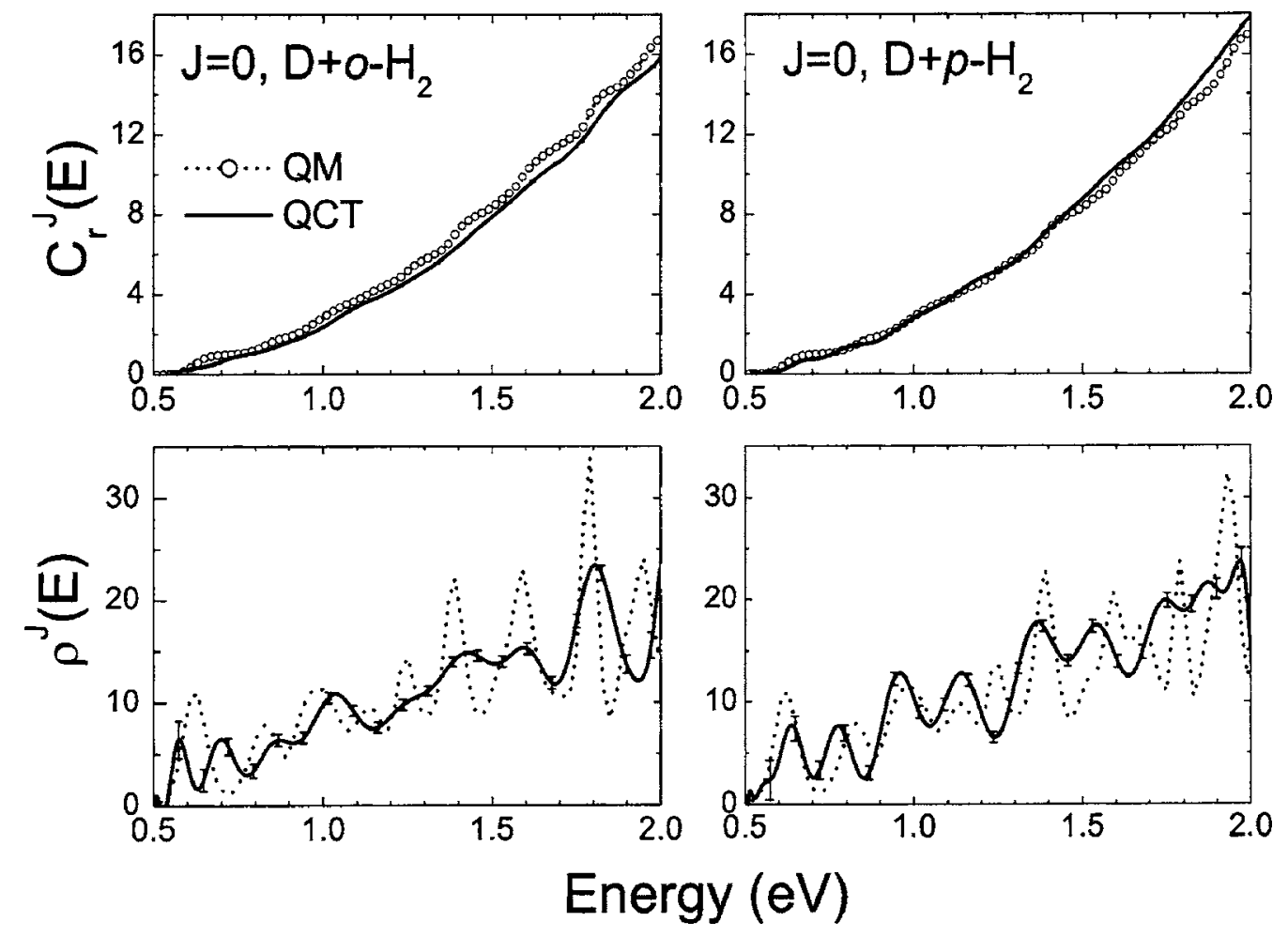

FIG. 9. Comparison of QM (open circles, dashed line) and QCT (solid line) $C_{r}^{J}(E)$ (top) and $\rho_{r}^{J}(E)$ (bottom) as a function of total energy for D $+o-\mathrm{H}_{2}$ and $\mathrm{D}+p-\mathrm{H}_{2}$ for $J=0$.

in the QM CRPs for ortho and para hydrogen are still very small, and they do not affect the rate constant values, even at temperatures above $1500 \mathrm{~K}$.

Figure 9 illustrates the larger differences that are observed in the QCT calculations between the reactivities of ortho and para hydrogen, with (even $j$ only) $p-\mathrm{H}_{2}$ being consistently more reactive than (odd $j$ only) $o-\mathrm{H}_{2}$. Note that the QCT CRP for $o-\mathrm{H}_{2}$ is systematically below the QM one, while that for $p-\mathrm{H}_{2}$ is always above. The comparison between the QM and QCT DRSs reveals some similarities, with some coincidence in the location of their respective peaks.

While the maxima observed in the QM DRS can be assigned to the opening of new internal states in the triatomic transition complex, ${ }^{5-7,11,12}$ this cannot be strictly the case in the QCT calculations, since no quantization exists outside the reactants. The $\mathrm{H}+\mathrm{H}_{2}$ reaction is to a large extent vibrationally adiabatic in the QM calculations, ${ }^{7,12,45}$ and it would appear that analogous behavior must be responsible for the structure observed in the QCT case, in spite of the fact that the internal energy of the transition state and the product is not quantized. The similar positioning of the maxima in the DRS seems to suggest that, although there can be no single QCT "transition state" for a given $v j \rightarrow v^{\prime} j^{\prime}$ reaction, there exists a narrow range of transition state internal energies through which the reaction is more likely to proceed. The QM peaks themselves are also broadened by an analogous process - there exists a region of nonadiabatic behavior on either side of the transition state, so that there is no single $v j \rightarrow\left[v_{s s} v_{b}^{K}\right]^{\ddagger} \rightarrow v^{\prime} j^{\prime}$ pathway through which the reaction exclusively progresses. ${ }^{12}$ Incorporation of these extra reaction pathways leads to a broadening of the maxima displayed in the QM DRS. While, in principle, there could be a continuum of possible paths for a given $v j \rightarrow v^{\prime} j^{\prime}$ reaction in the QCT case, the presence of the maxima in $\rho_{r}^{J}(E)$ seems to suggest that a quasiclassical analog to the QM bottlenecks does exist, indicating some degree of vibrational adiabaticity. In fact, in a series of recent works, Skodje and co-workers ${ }^{12,13,45}$ have emphasized the role of the assumption of adiabatic separability along the reaction coordinate with respect to the quantized internal modes of the complex, i.e., with respect to the quantum bottlenecks. They derived a model using a Frank-Condon approximation that allows computation of the $S$ matrix using a local transition state dynamics. The results obtained with this approximation were in good agreement with the exact results. If, in the adiabatic approximation, each internal mode of the reactive complex is assumed to conserve the action along a reaction coordinate, an approximation which seems to work very well in the QM treatment, it is very likely that a similar situation will also be obtained in the classical approach.

If the maxima in the QCT DRS can be considered "quasiclassical bottlenecks," it is pertinent to ask whether or not are they associated with some specific property. In quantum mechanics the bottlenecks are associated with short-lived species and, in fact, calculations of their lifetimes have been presented for the $\mathrm{H}+\mathrm{H}_{2}$ reaction. $^{7}$ The passage over a barrier is usually associated with a time delay, due to the slowing down of the system as it crosses the top of barrier. This interpretation was used to explain the time delay occurring in the forward scattering direction for the various isotopic variants of the $\mathrm{H}_{3}$ reaction. ${ }^{31,46}$ The time delays obtained in classical calculations ${ }^{47}$ coincide neatly with those obtained with 


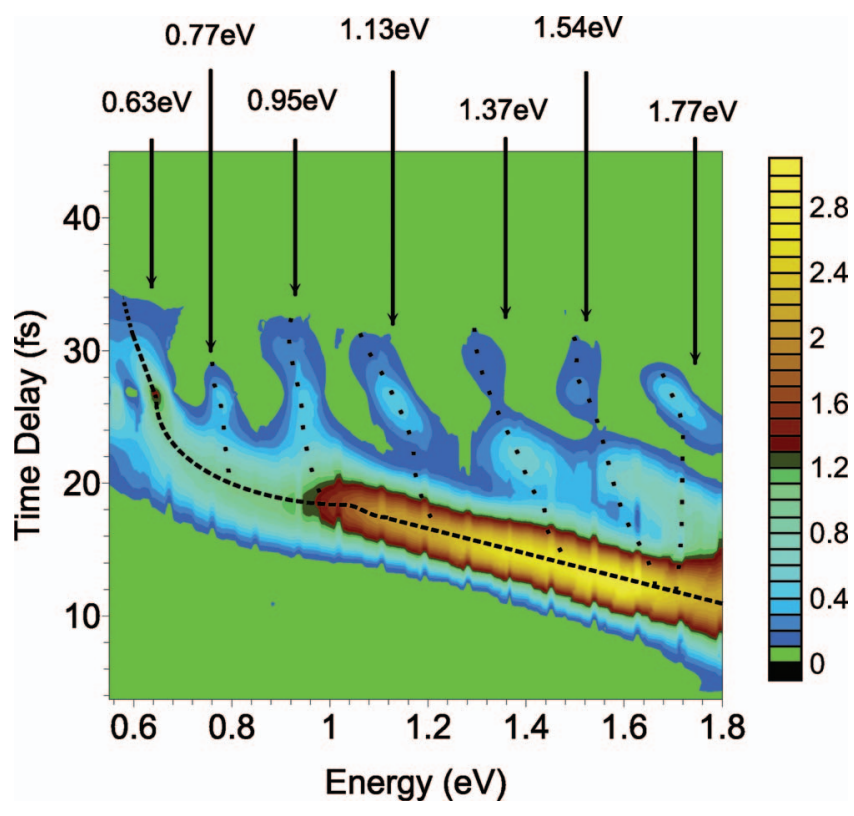

FIG. 10. (Color) Contour plot of the density of reactive states as a function of total energy and collision time, $\rho^{J=0}(E, \tau)$, for the $\mathrm{D}+p-\mathrm{H}_{2}$ reaction. The arrows labeled with energy values correspond to the various maxima in the $J=0$ DRS shown in Fig. 9.

the plane wave packet analysis of the QM time-dependent scattering $^{48}$ and with time-independent calculations. ${ }^{49}$

In the present work, collision times $\tau$ have also been analyzed to ascertain whether the various maxima (minima) in the DRS are associated with relatively longer (shorter) collision times. The distribution of collision times for reactive trajectories was calculated at different energies, in particular, for those corresponding to the various maxima and minima in the $J=0 \mathrm{DRS}$. As expected, the most probable collision time decreases with the total energy, first rapidly and then more slowly. Superimposed on this trend, the distributions associated with the maxima in the DRS are slightly, but noticeably, broader than those corresponding to the minima, and more interestingly, they have a longer tail extending to higher values of $\tau$. Information about the energy and collision time dependence of the DRS, $\rho_{r}^{J=0}(E, \tau)$, can be compactly presented using a contour plot (see the Appendix), as shown in Fig. 10 for the $\mathrm{D}+p-\mathrm{H}_{2}$ reaction. Integration of this function over $\tau$ yields the usual $\rho_{r}^{J=0}(E)$. The most probable collision time, shown in the figure as a dashed line, decreases with total energy, and ranges from $\approx 35 \mathrm{fs}$ at $E$ $=0.6 \mathrm{eV}$ to $\approx 15 \mathrm{fs}$ at $1.8 \mathrm{eV}$. This evolution of the most probable $\tau$ with $E$ constitutes the main feature of the double distribution, and the $\rho_{r}^{J=0}(E, \tau)$ along this ridge increases almost monotonically with $E$ without displaying evident maxima or minima. However, in addition to this dominant feature, a series of smaller ridges is also observed, as indicated in the figure by the dotted lines protruding from the main ridge. It would appear that there are some energy intervals over which the distribution of collision times extends to larger values of $\tau$. These energies correspond quite accurately with those of the maxima in $\rho_{r}^{J=0}(E)$. Furthermore, the separation between these tails $(\sim 0.2 \mathrm{eV})$ is quite close to both the transition state symmetric stretching frequency in $\mathrm{DHH}(0.26 \mathrm{eV})$ and to two quanta in the bend $(0.23 \mathrm{eV})$.
The latter mode, in particular, seems to be an important feature in the assigned structures of the QM DRS for $\mathrm{D}+\mathrm{H}_{2}{ }^{11}$ The tails in $\rho_{r}^{J=0}(E, \tau)$, once integrated over $\tau$, give rise to the observed maxima in the DRS. Given that the above analysis for both diatomic parities yielded similar results, it can be concluded that the presence of maxima in the DRS is associated with the existence of reactive trajectories with somewhat longer collision times or, at least, with a distribution of $\tau$ biased towards longer values.

The association of the observed maxima in the QCT DRS with a broader distribution of collision times is perhaps not surprising. It suggests a possible connection with periodic orbits which Pollak and co-workers studied in the collinear $\mathrm{H}_{3}$ reaction. ${ }^{50-52}$ They demonstrated that the best choice of transition state in a classical collinear collision is a classical bound state embedded in the continuum, which is necessarily a periodic orbit. Pollak and Child ${ }^{52}$ showed that collinear resonances can be described classically by a resonant energy transfer between adiabatic states of the incoming and outgoing diatoms, as long as there exists a well in the adiabatic PES, which usually shows up in the vicinity of adiabatic thresholds of new vibrational channels. By the semiclassical quantization of the action along the periodic orbit it was possible to predict very accurately the location of the resonances found in QM calculations. More recently Sadhegi and Skodje ${ }^{53}$ carried out a comparative analysis of the collinear $\mathrm{D}+\mathrm{H}_{2}$ reaction using spectral quantization methods and a semiclassical treatment based on periodic orbits. They concluded that most resonances are associated with single quantized periodic orbits in the transition state region. The situation is considerably more complex in 3D collisions, but it has been shown that the adiabatic barrier maxima correspond to quantized quasiperiodic trajectories (see Refs. 7 and 51 and references therein). It will be interesting to perform a detailed analysis of the trajectories with longer $\tau_{\text {col }}$ pertaining to the ridges shown in Fig. 10.

As mentioned above, the only quantization in the present QCT calculations is in the internal energy of the reactants, the total angular momentum $J$, and the orbital angular momentum $l$. Any structure in the $C_{r}^{J}(E)$ must arise from the initial state quantization. In the absence of reactant quantization, one would expect a completely smooth increase in $C_{r}^{J}(E)$ with energy. Better agreement between QM and QCT results is therefore likely if the energy level spacing of the reagents, products, and transition states was considerably smaller.

Figure 11 investigates the effect of varying the spacing of the internal energy levels of the reactants on the QM and QCT $C_{r}^{J=0}(E)$ for $\mathrm{H}+\mathrm{H}_{2}$. The spacing is reduced by a given factor by decreasing the value of Planck's constant $h$ by the same amount (note that the density of internal states scales with the square of this factor). QCT and QM CRPs calculated with a modified Planck constant $h^{\prime}=h / \sqrt{10}$ are represented in Fig. 11. By comparing with the results of Fig. 3, it is clear that the absolute value of the CRP has increased by a factor of 10. More importantly, the agreement between QM and QCT is noticeably better than for $h^{\prime}=h$, as the QM CRP becomes closer to a smooth curve. Additional QCT calculations were carried out for $h^{\prime \prime}=h / 10$, and the CRP is also 

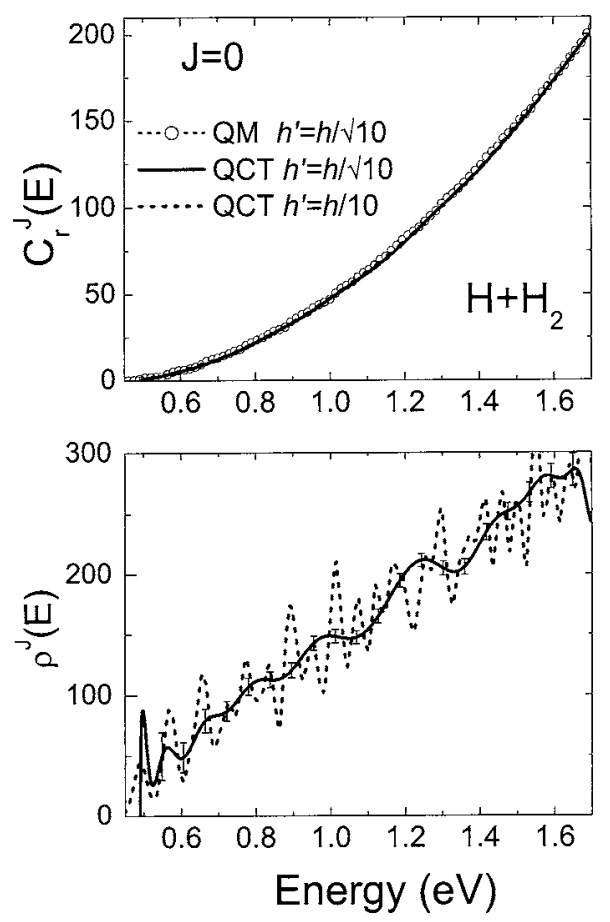

FIG. 11. Comparison of QM (open circles, dashed line) and QCT (solid line) $C_{r}^{J}(E)$ (top) and $\rho_{r}^{J}(E)$ (bottom) for $\mathrm{H}+\mathrm{H}_{2}, J=0$, with a modified value of Planck's constant, $h^{\prime}=h / \sqrt{10}$. The QCT CRP with $h^{\prime \prime}=h / 10$ is also represented on the top panel divided by 10 to be directly comparable to the results for $h^{\prime}=h / \sqrt{10}$.

included in the top panel of the figure, divided by 10 so as to be plotted on the same scale. The agreement between the CRP with these two scaling factors for $h$ is almost perfect, indistinguishable within the resolution of the figure, and clearly shows that the magnitude of CRP scales with the square of the quotient, $h^{\prime} / h^{\prime \prime}$. As a result of the energy spacing being smaller, the steplike structure in the $C_{r}^{J}(E)$ becomes much more diffuse. This can be rationalized by considering the density of internal states of the triatomic transition state. As $h^{\prime}$ becomes smaller, the number of internal states per unit energy increases, and so the steps in $C_{r}^{J}(E)$ become both more frequent and smaller in relative magnitude, accounting for the smoother increase in $C_{r}^{J}(E)$. The bottom panel shows this nicely, comparing QCT and QM $\rho_{r}^{J=0}(E)$ for $h^{\prime}=h / \sqrt{10}$. In both cases the structure is much less significant when compared with the absolute value of $\rho_{r}^{J=0}(E)$. Note that, because the zero point energy of the reactants scales with $h^{\prime}$, the QM and QCT energy thresholds for reaction also decrease with $h^{\prime}$, and in the limit of $h \rightarrow 0$, will be just the height of the barrier. Additionally, the effect of tunneling and zero point energy becomes smaller as $h$ decreases.

\section{CONCLUSIONS}

In this work, we have presented a general quasiclassical trajectory method to calculate cumulative reaction probabilities (CRPs). It is based on a discrete sampling of integer values of the total, $J$, and orbital, $l$, angular momentum quantum numbers in strict correspondence with that used in QM calculations. This procedure allows the determination of the CRP at a given $J$, or that summed over all $J$ contributing to the reaction, i.e., the total CRP. By using this latter magnitude, rate constants can be readily and effectively determined.

To test the method and to check the reliability of the QCT results, extensive calculations of the CRP have been carried out at a given $J$ for several isotopic variants of the $\mathrm{H}_{3}$ exchange reaction. The results have been compared with exact and thorough QM calculations, which include results for all $J$ values in a dense grid of total energies from 0.25 to $1.65 \mathrm{eV}$. It has been found that QCT $J$-resolved CRPs are in excellent agreement with the corresponding QM results, with two exceptions. One is their values at the lowest collision energies, where, as could be expected, tunneling plays an important role. While the QCT CRPs are zero, the QM CRPs at low energies, although small in absolute terms, become significant, and this is neatly reflected in the values of the rate constant. The other exception is the steplike structure that appears in the QM CRP for $J=0$, which is washed out in the QCT calculations. These structures have been traced back to quantum transition state features or dynamical bottlenecks which gate the flow of reactive flux from reactants to products.

The manifestation of these quantized transition states can be best appreciated if the density of reactive states (DRS), that is, the derivative of the CRP with respect to the energy, is represented as a function of the energy. A series of pronounced peaks shows up in what constitutes an energy level spectrum of the transition state, that can be assigned using the conventional model of a linear triatomic complex with a missing degree of freedom. Interestingly, the QCT DRS also displays some structure that is less pronounced and smoother than those found in the QM DRS. The locations of the relatively broad peaks in the QCT DRS roughly coincide with those found in the corresponding QM DRS. A collision time analysis of the trajectories pertaining to the successive maxima and minima of the DRS was performed, and showed that the distributions of collision times of the former were somewhat broader than those of the latter, with a tail extending to higher values of the collision time. Trajectories corresponding to maxima therefore have on average very slightly longer collision times than those appearing in the adjacent minima. This effect is relatively small, but it is appreciable. In consequence, it is possible to associate the peaks in the QCT DRS with the existence of classical bottlenecks. The situation is somewhat analogous to that occurring in forward scattering, in which the surmounting of the centrifugal barrier causes a delay in the appearance of products after the reactive collision.

In order to further assess the convergence of the QM and QCT CRPs in the correspondence limit, QM and QCT calculations for $J=0$ were carried out using a Planck constant $h^{\prime}=h / \sqrt{10}$. The CRP scales with $\left(h^{\prime} / h\right)^{2}$ and, as expected, it was found that the agreement between QM and QCT results improves noticeably. Some oscillatory behavior of the QM DRS still persists, but becomes gradually closer to the QCT case as $h^{\prime}$ is reduced.

Finally, the dynamics of various isotopic variants of the $\mathrm{H}_{3}$ exchange reactions have been discussed by comparing the exact QM and QCT CRPs. Many of the findings have been 
discussed in other contexts, but it is the first time, to the best of our knowledge, that such joint analysis of no less than four isotopic variants of the title reaction has been carried out.

\section{ACKNOWLEDGMENTS}

Three of the authors (F.J.A., V.S.R., and J.F.C.) acknowledge funding by the Spanish Ministry of Education and Science (Grant No. CTQ2005-08493-C01-01). One of the authors (M.B.) acknowledges EPSRC for financial support. One of the authors (F.J.A.) gratefully acknowledges the funding of the University Complutense under the program "Sabáticos Complutense." Another author (J.F.C.) would like to thank the support from Program Ramón y Cajal.

\section{APPENDIX: CALCULATION OF $C_{r}^{J}(E)$ AND $C_{r}(E)$ USING AN EXPANSION IN LEGENDRE POLYNOMIALS}

The method for calculating the CRP described in Sec. II assumes a single value of the total energy. Hence to determine the CRP at a sufficiently dense grid of energies, it is necessary to run many batches of trajectories each at a different value of $E$ and $J$.

An alternative method, which has been employed in the calculation of cross sections as a function of collision energy, ${ }^{42,43,54}$ consists of running trajectories at a given $J$ sampling the total energy randomly and uniformly within the interval $\left[E_{1}, E_{2}\right]$, such that the total energy threshold will be $E_{0} \geqslant E_{1}$. Once the value of $E$ is fixed for each trajectory, the total number of energetically accessible states, $n(E)$, is determined and the initial $v, j$ state is randomly selected with equal probability from that set of states. The rest of initial conditions are selected as in Ref. 30. Then the CRP as a function of $E$ can be fitted to a series of Legendre polynomials. The method is entirely analogous to that used for calculating reaction probabilities as a function of the energy for a given $J$ value, $P_{r}^{J}(E)$ (see Ref. 30).

It is easy to show that $C_{r}^{J}(E)$, when the energy is continuously scanned, can be written in terms of a series of Legendre polynomials as

$$
C_{r}^{J}(E)=\frac{2 Q}{E_{\max }-E_{\min }} \sum_{m=0}^{M} a_{m} P_{m}[x(E)],
$$

where $P_{n}(x)$ is the $n$th degree Legendre polynomial whose argument is the reduced variable $x(E)$ defined in $[-1,1]$, and the normalization constant $Q$ is given by

$$
Q=\frac{S_{r}}{N(J)}\left(E_{2}-E_{1}\right),
$$

where $N(J)$ is the total number of trajectories run in the interval $\left[E_{1}, E_{2}\right]$ and $S_{r}$ is the sum of the weights $w_{i}$ of each individual reactive trajectory, i.e.,

$$
S_{r}=\sum_{i=1}^{N_{r}} w_{i}
$$

$$
w_{i}=\left[2 \min \left(J_{i}, j_{i}\right)+1\right] n\left(E_{i}\right),
$$

with $E_{i}, J_{i}$, and $j_{i}$ the total energy and the total and rotational angular momentum quantum numbers, respectively, for the $i$ th reactive trajectory. The normalization constant $Q$ is nothing but the integral of $C_{r}^{J}(E)$ in the $\left[E_{1}, E_{2}\right]$ energy interval.

This method offers significant advantages over that using discrete values of total energies, avoiding the necessity of running many batches of trajectories at different energies. In addition, the derivatives of the $C_{r}^{J}(E)$, the DRS, $\rho_{r}^{J}(E)$, are analytical and can be readily determined. It is also quite straightforward to calculate the statistical errors by propagation of those associated with the $a_{m}$ coefficients.

A similar method can be applied to calculate the total CRP in a single batch of trajectories by scanning the total energy continuously within an interval $\left[E_{1}, E_{2}\right]$. Once the value of $E$ is randomly sampled, the $J$ value is obtained by a sampling proportional to the number of projections $2 J+1$.

The expression of $C_{r}(E)$ in terms of a series of Legendre polynomials is similar to Eq. (A1) but now the normalization factor is given by $Q=\left(E_{2}-E_{1}\right) S_{r} / N$, where $N$ is the total number of trajectories and the weights for reactive trajectories is now given by

$$
w_{i}=\left[2 \min \left(J_{i}, j_{i}\right)+1\right] n\left(E_{i}\right)\left[J_{\max }\left(E_{i}\right)+1\right]^{2},
$$

where $J_{\max }\left(E_{i}\right)$ is the maximum value of $J$ at which reaction can take place at $E_{i}$.

The sampling method to determine the total CRP can be effectively used to calculate directly the rate constants $k(T)$ without needing to determine first the CRP and avoiding the numerical integration.

It is straightforward to demonstrate that the value of $k(T)$ at a given temperature must be

$$
k(T)=\frac{E_{2}-E_{1}}{N} \frac{\tilde{S}_{r}}{h \Phi_{\text {rel }}(T) Q_{v j}^{B C}(T)},
$$

where $\Phi_{\text {rel }}(T)$ and $Q_{v j}^{B C}(T)$ are the translational and internal partition functions of the reactants. $\widetilde{S}_{r}$ is given by

$$
\begin{aligned}
& \widetilde{S}_{r}=\sum_{i=1}^{N_{r}} \widetilde{w}_{i}, \\
& \widetilde{w}_{i}=\left[2 \min \left(J_{i}, j_{i}\right)+1\right] n\left(E_{i}\right)\left[J_{\max }\left(E_{i}\right)+1\right]^{2} \exp \left(-E_{i} / k_{B} T\right),
\end{aligned}
$$

where $N$ and $N_{r}$ are the total number and the number of reactive trajectories in the interval $\left[E_{1}, E_{2}\right]$. In the case of reaction with homonuclear molecules, the nuclear spin weights $I_{i}\left(2 I_{i}+1\right)$ or $\left(I_{i}+1\right)\left(2 I_{i}+1\right)$ for each trajectory can also be introduced in Eq. (A8).

As mentioned in Sec. II, collision times were calculated for batches of trajectories at $J=0$. In order to obtain the dependence of the CRP and DRS as a function of both total energy and collision time, the results were fitted to a double series of Legendre polynomials, similarly to that previously used for the probability density function of other variables. ${ }^{54}$ 
Once the double dependent $C_{r}^{J}(E, \tau)$ is obtained in terms of a series of Legendre polynomials, the DRS dependent on $E$ and $\tau, \rho_{r}^{J}(E, \tau)$, is simply given by derivation with respect to $E$.

${ }^{1}$ W. H. Miller, J. Chem. Phys. 62, 1899 (1975).

${ }^{2}$ W. H. Miller, J. Chem. Phys. 65, 2216 (1976).

${ }^{3}$ W. H. Miller, Acc. Chem. Res. 9, 306 (1976).

${ }^{4}$ W. H. Miller, Acc. Chem. Res. 26, 174 (1993).

${ }^{5}$ D. C. Chatfield, R. S. Friedman, D. G. Truhlar, B. C. Garrett, and D. W. Schwenke, J. Am. Chem. Soc. 113, 486 (1991).

${ }^{6}$ D. C. Chatfield, R. S. Friedman, D. G. Truhlar, and D. W. Schwenke, Faraday Discuss. Chem. Soc. 91, 289 (1991).

${ }^{7}$ D. C. Chatfield, R. S. Friedman, D. W. Schwenke, and D. G. Truhlar, J. Phys. Chem. 96, 2414 (1992).

${ }^{8}$ In the literature the CRP is commonly denoted as $N^{J}(E)$. In this work we have reserved $N$ and $N_{r}$ for the total number of trajectories and the number of reactive trajectories at a given total energy $E$ and total angular momentum $J$. In order to avoid confusion with this notation, we will use $C_{r}^{J}(E)$ and $C_{r}(E)$ for the cumulative reaction probabilities at a given $J$ and for the total CRP, respectively.

${ }^{9}$ W. H. Miller, J. Chem. Phys. 61, 1823 (1974).

${ }^{10}$ S. Chapman, S. M. Hornstein, and W. H. Miller, J. Am. Chem. Soc. 97, 892 (1975).

${ }^{11}$ D. C. Chatfield, S. L. Mielke, T. C. Allison, and D. G. Truhlar, J. Chem. Phys. 112, 8387 (2000)

${ }^{12}$ R. T. Skodje and X. Yang, Int. Rev. Phys. Chem. 23, 253 (2004).

${ }^{13}$ D. Dai, C. C. Wang, S. A. Harich, X. Wang, X. Yang, S. D. Chao, and R. T. Skodje, Science 300, 1730 (2003).

${ }^{14}$ G. C. Schatz, J. Chem. Phys. 90, 3582 (1989).

${ }^{15}$ G. C. Schatz, J. Chem. Phys. 90, 4847 (1989).

${ }^{16}$ J. F. Castillo, D. E. Manolopoulos, K. Stark, and H.-J. Werner,J. Chem. Phys. 104, 6531 (1996).

${ }^{17}$ T. Xie, D. Wang, J. Bowman, and D. E. Manolopoulos, J. Chem. Phys. 116, 7461 (2002).

${ }^{18}$ Y. Lin, B. Ramachandran, K. Nobusada, and H. Nakamura, J. Chem. Phys. 113, 1018 (2000)

${ }^{19}$ Y. Lin, B. Ramachandran, K. Nobusada, and H. Nakamura, J. Chem. Phys. 114, 1549 (2001)

${ }^{20}$ W. H. Miller, S. D. Schwartz, and J. W. Tromp, J. Chem. Phys. 79, 4889 (1983).

${ }^{21}$ T. Seideman and W. H. Miller, J. Chem. Phys. 96, 4412 (1992).

${ }^{22}$ T. Seideman and W. H. Miller, J. Chem. Phys. 97, 2499 (1992).

${ }^{23}$ U. Manthe and W. H. Miller, J. Chem. Phys. 99, 3411 (1993).

${ }^{24}$ P. N. Day and D. G. Truhlar, J. Chem. Phys. 94, 2045 (1991).

${ }^{25}$ U. Manthe, T. Seideman, and W. H. Miller, J. Chem. Phys. 99, 10078 (1993).

${ }^{26}$ U. Manthe, T. Seideman, and W. H. Miller, J. Chem. Phys. 101, 4759
(1994).

${ }^{27}$ F. Huarte-Larraaga and U. Manthe, J. Chem. Phys. 113, 5115 (2000).

${ }^{28}$ F. Huarte-Larraaga and U. Manthe, J. Chem. Phys. 117, 4635 (2002).

${ }^{29}$ A. Chakraborty and D. G. Truhlar, Proc. Natl. Acad. Sci. U.S.A. 102, 6744 (2005)

${ }^{30}$ F. J. Aoiz, V. Sáez-Rábanos, B. Martínez-Haya, and T. González-Lezana, J. Chem. Phys. 123, 094101 (2005).

${ }^{31}$ F. J. Aoiz, L. Bañares, and V. J. Herrero, Int. Rev. Phys. Chem. 24, 119 (2005).

${ }^{32}$ T. Gónzalez-Lezana, A. Aguado, M. Paniagua, and O. Roncero, J. Chem. Phys. 123, 194309 (2005).

${ }^{33}$ S. L. Mielke, G. C. Lynch, D. G. Truhlar, and D. W. Schwenke, Chem. Phys. Lett. 216, 441 (1993).

${ }^{34}$ S. L. Mielke, G. C. Lynch, and D. G. Truhlar, J. Phys. Chem. 98, 8000 (1994).

${ }^{35}$ A. I. Boothroyd, W. J. Keogh, P. G. Martin, and M. R. Peterson, J. Chem. Phys. 104, 7139 (1996).

${ }^{36}$ J. Vanícek, W. H. Miller, J. F. Castillo, and F. J. Aoiz, J. Chem. Phys. 123, 054108 (2005).

${ }^{37}$ D. Skouteris, J. F. Castillo, and D. E. Manolopoulos, Comput. Phys. Commun. 133, 128 (2000).

${ }^{38}$ G. W. Johnston, H. Kornweitz, I. Schechter, A. Persky, B. Katz, R. Bersohn, and R. D. Levine, J. Chem. Phys. 94, 2749 (1991).

${ }^{39}$ F. J. Aoiz, L. Bañares, V. J. Herrero, V. Sáez Rábanos, K. Stark, and H.-J. Werner, J. Chem. Phys. 102, 9248 (1995).

${ }^{40}$ N. C. Blais, D. G. Truhlar, and B. C. Garret, J. Chem. Phys. 78, 2363 (1983).

${ }^{41}$ L. Bañares and M. J. D’Mello, Chem. Phys. Lett. 277, 465 (1997).

${ }^{42}$ F. J. Aoiz, L. Bañares, T. Díez-Rojo, V. J. Herrero, and V. Sáez Rábanos, J. Phys. Chem. A 100, 4071 (1996).

${ }^{43}$ F. J. Aoiz, L. Bañares, V. J. Herrero, V. Sáez Rábanos, and I. Tanarro, J. Phys. Chem. A 101, 6165 (1997).

${ }^{44}$ S. L. Mielke, K. A. Peterson, D. W. Schwenke, B. C. Garrett, D. G. Truhlar, J. V. Michael, M.-C. Su, and J. W. Sutherland, Phys. Rev. Lett. 91, 063201 (2003)

${ }^{45}$ M. Gustafsson and R. T. Skodje, J. Chem. Phys. 124, 144311 (2006)

${ }^{46}$ D. E. Manolopoulos, Nature (London) 419, 266 (2002).

${ }^{47}$ F. J. Aoiz, V. J. Herrero, and V. Sáez Rábanos, J. Chem. Phys. 97, 7423 (1992).

${ }^{48}$ S. C. Althorpe, J. Chem. Phys. 117, 4623 (2002).

${ }^{49}$ S. A. Harich, D. Dai, C. C. Wang, X. Yang, S. D. Chao, and R. T. Skodje, Nature (London) 419, 281 (2002).

${ }^{50}$ E. Pollak and P. Pechukas, J. Chem. Phys. 69, 1218 (1978)

${ }^{51}$ E. Pollak, Chem. Phys. Lett. 91, 27 (1982).

${ }^{52}$ E. Pollak and M. S. Child, Chem. Phys. 60, 23 (1981).

${ }^{53}$ R. Sadhegi and R. T. Skodje, J. Chem. Phys. 102, 193 (1995).

${ }^{54}$ F. J. Aoiz, L. Bañares, and V. J. Herrero, J. Chem. Soc., Faraday Trans. 94, 2483 (1998). 